EPJ Web of Conferences 41, 10016 (2013)

DOI: $10.1051 /$ epjconf/20134110016

C Owned by the authors, published by EDP Sciences, 2013

\title{
Ultrafast time resolved reflection high energy electron diffraction with tilted pump pulse fronts
}

\author{
P. Zhou, C. Streubühr, A. Kalus, T. Frigge, S. Wall, A. Hanisch-Blicharski, \\ M. Kammler, M. Ligges, U. Bovensiepen, D. von der Linde and M. Horn-von Hoegen \\ Department of Physics \& Center for Nanointegration CeNIDE, University of Duisburg-Essen, \\ Lotharstr. 1, 47048 Duisburg, Germany \\ E-mail: ping.zhou@uni-due.de
}

\begin{abstract}
We present time-resolved RHEED from a laser excited $\mathrm{Pb}(111)$ surface using a pulse front tilter for the compensation of the velocity mismatch of electrons and light. The laser pulses with tilted fronts were characterized by a spatially resolving cross correlator. The response of the surface upon excitation was observed to be less than 2 ps.
\end{abstract}

Ultrafast electron diffraction is a promising technique for studying the dynamics of the atomic structure on a sub-picosecond time scale. Time resolved transmission electron diffraction (TR-TED) has been used, for example, to study bulk phenomena such as ultrafast laser-induced structural phase transitions [1], cooperative rearrangement of crystalline structures [2], and dynamics of charge density waves [3]. Time resolution of less than $100 \mathrm{fs}$ [4] has been achieved. Time resolved reflection high energy electron diffraction (TR-RHEED), on the other hand, has been used for the investigation of ultrafast surface dynamics, for example the observation of ultrafast heating of surfaces [5], the heat transfer in hetero structures [6] and laser-induced phase transition of the surface reconstructions [7]. However, the time resolution in these experiments was limited to tens of picoseconds because of the different velocities of electrons and light in combination with the grazing angle of incidence of the electron beam in RHEED geometry.

This velocity mismatch can be overcome and the time resolution significantly improved by tilting the laser pulse front with respect to the phase fronts. Baum et al. [8] have demonstrated a configuration for pulse front tilting and achieved an improvement of the temporal resolution to less than one picosecond. Here we report the use of a $4 \mathrm{f}$ zero dispersion delay line capable of matching the pulse front of the laser to the electron pulse. The improvement of the temporal resolution is demonstrated by measuring the ultrafast lattice heating of $\mathrm{Pb}(111)$ islands on a $\mathrm{Si}(111)$ surface.

Generally speaking, when a laser pulse encounters angular dispersion, the planes of constant amplitude (intensity) will be tilted with respect to the planes of constant phase, for example when the pulse is reflected from a diffraction grating [9]. The pulse then broadens during the free propagation after the dispersive component. The broadening is reversed and the original pulse width restored by passing the pulse through the two lenses in a $4 \mathrm{f}$ configuration. Our pulse tilter was designed to compensate the velocity mismatch for $30 \mathrm{keV}$ electrons. Based on the results of our electron source [10] a temporal resolution of better than $700 \mathrm{fs}$ is expected.

In order to independently control and characterize the tilted pulse we used a cross-correlator based on second harmonic generation ( $\mathrm{SHG}$ ). The special feature of this cross-correlator is the possibility to perform spatially resolved measurements of the cross-correlation function between the

This is an Open Access article distributed under the terms of the Creative Commons Attribution License 2.0, which permits unrestricted use, distribution, and reproduction in any medium, provided the original work is properly cited. 
original and the tilted pulses. The original beam was focussed and overlapped with the tilted beam at different positions across the beam width as shown in Fig. 1a. In order to determine the tilt angle we measured the difference in the temporal overlap of the two pulses at different positions. The widths of the correlation function at different positions maps out the pulse duration across the beam.

In our experiment the laser pulses were generated by a Ti:Sapphire CPA system with $50 \mathrm{fs}$ duration at $800 \mathrm{~nm}$ wavelength and $5 \mathrm{kHz}$ repetition rate. A small fraction of the laser beam was converted to the third harmonic in order to generate photoelectrons for the electron source. The electrons were accelerated to $30 \mathrm{keV}$ and detected by a MCP after diffraction under grazing incidence. The main part of the laser beam was split to form the delayed probe pulse for the crosscorrelator and, after having passed the tilter, the actual pump pulse. For measuring purposes the tilted pump pulses could alternatively be sent to a BBO crystal in the cross-correlator placed at a position equivalent to the sample. The grating had 2000 lines $/ \mathrm{mm}$ and a reflectivity of about $90 \%$ at $800 \mathrm{~nm}$. For an angle of incidence on the grating of $52^{\circ}$ a tilt angle of $71^{\circ}$ was calculated. Fig. $1 \mathrm{~b}$ shows the cross-correlation between the unmodified laser pulses and the tilted laser pulses at three different positions as marked by a circle, a triangle and a square in Fig. 1a. From the difference of the temporal overlap at different positions across the laser beam - in our case about $0.52 \mathrm{~mm}$ from circle to square positions in Fig. 1a - we determined a tilt angle of $72^{\circ}$. The full width at half maximum (FWHM) of the correlation function is determined by the widths of the two pulses plus a contribution due to beam geometry effects.

a)
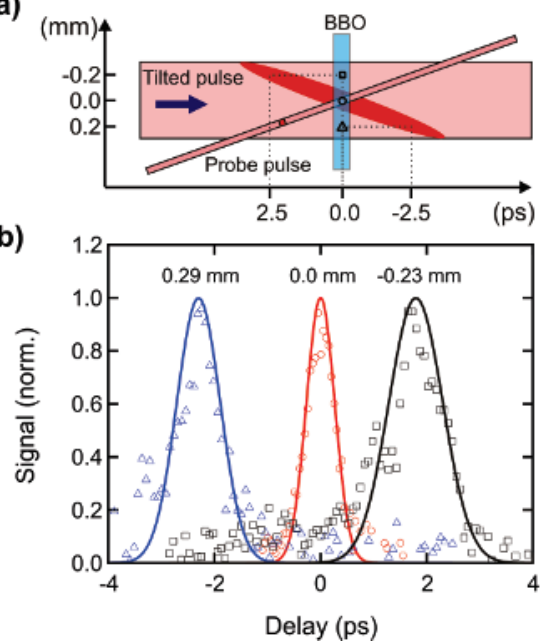

c)

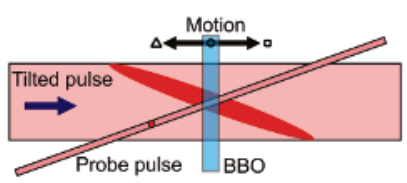

d)

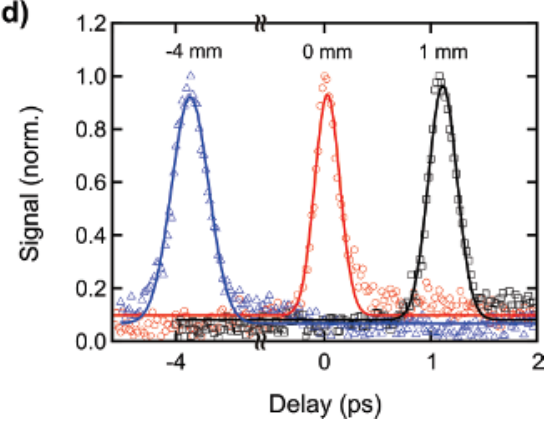

Fig. 1: a) Scheme of the beam geometry in the SHG crystal (cross-correlator). b) Crosscorrelations between unmodified and tilted laser pulses at three different positions across the beam. The difference of the distance between the circle and the square is $0.52 \mathrm{~mm}$ which gives a tilt angle of about $72^{\circ}$. c) Scheme of the BBO crystal displacement. d) Cross-correlations between unmodified and tilted laser pulses at different $\mathrm{BBO}$ positions.

With this configuration the original pulse can be recompressed at a plane corresponding to an image of the grating surface. Any deviation from this plane leads to a broadening of the pulse duration. In the TR-RHEED experiment normal incidence of the pump beam is used and therefore the angle between the sample surface and the grating image is approximately equal to the tilt angle leading to a broadening of the pulse duration across the sample surface of about $300 \mathrm{fs}$. From the correlation function we obtained a FWHM of about $600 \mathrm{fs}$ over a range of $2 \mathrm{~mm}$ which indicated that the duration of the tilted pulse is less than that value. The pulse duration increases when the BBO moves away from its optimal position (Fig. 1d). The pulse duration was measured to be within 1 ps by a deviation of $4 \mathrm{~mm}$ from the optimal position. 
The tilted laser pulse was used as pump pulse in TR-RHEED experiments on $\mathrm{Pb}(111)$ islands grown on a $\mathrm{Si}(111)$ substrate by deposition of $\mathrm{Pb}$ at room temperature. The $\mathrm{Pb}$ forms 3-dimensional islands upon a disordered $\mathrm{Pb}$ wetting layer on the $\mathrm{Si}(111)$ surface. The islands are atomically flat with a width of few $10 \mu \mathrm{m}$ [11]. At $3^{\circ}$ angle of incidence of the electron beam, two strong diffraction spots were observed as depicted in the inset of Fig. 2b. The change of intensity of the (00) spot observed after laser excitation is plotted in Fig. 2b. We attribute the intensity drop to the transient Debye-Waller effect, indicating the heating of the lattice caused by the pump pulse.

The dashed and solid lines depict exponential fits. In order to optimize the tilter geometry we varied the imaging of the grating. The fastest response of 3 ps was obtained when the center of the grating was imaged onto the sample. The observed time constant of $3 \mathrm{ps}$ is the convolution of compensation limit of the tilter and the response of the $\mathrm{Pb}$ lattice. In fact, with a careful optimization of all experimental parameters we were able to observe an overall time constant of $1.8 \mathrm{ps}$.

The velocity mismatch between laser and electron pulse is proportional to the illuminated sample area. We expected an improvement of the temporal resolution if we reduce the sample area by laterally removing the sample out of the electron beam. In contrast, the experimental results showed the same time constants, suggesting that the actual material response time is responsible for the $1.8 \mathrm{ps}$ time constant and that the time resolution is better than this value.

This work was supported by the German Research Foundation DFG through the collaborative research center SFB 616 "Energy Dissipation at Surfaces".

[1] B. J. Siwick, J. R. Dwyer, R. E. Jordan and R. J. D. Miller, Science 302, 1382-85 (2003)

[2] P. Baum, D. S. Yang and A. H. Zewail, Science 318, 788 (2007)

[3] M. Eichberger, H. Schäfer, M. Krumova, M. Beyer, J. Demsar, H. Berger, G. Moriena, G. Sciaini and R. J. D. Miller Nature 468, 799 (2010)

[4] T. van Oudheusden, P. L. E. M. Pasmans, S. B. van der Geer, M. J. de Loos, M. J. van der Wiel and O. J. Luiten, Phys. Rev. Lett. 105, 264801 (2010)

[5] M. Aeschlimann, E. Hull, J. Cao, C. A. Schmuttenmaer, L. G. Jahn, Y. Gao, H. E. Elsayed-Ali, D. A. Mantell and M. R. Scheinfein, Rev. Sci. Instrum. 66, 1000 (1995)

[6] B. Krenzer, A. Janzen, P. Zhou, D. von der Linde and M. Horn-von Hoegen, New J. Phys. 8, 190 (2006)

[7] S. Möllenbeck, A. Hanisch-Blicharski, P. Schneider, M. Ligges, P. Zhou, M. Kammler, B. Krenzer and M. Horn-von Hoegen, MRS Proceedings 1230-MM03-09 (2009)

[8] P. Baum and A. H. Zewail, Proc. Natl. Acad. Sci. U.S.A. 103, 16105 (2006)

[9] Z. Bor, B. Rácz, G. Szabó, M. Hubert and H. A. Hazim, Optical Engineering 32, 2501 (1993)

[10] M. Ligges, I. Rajkovic, P. Zhou, O. Posth, C. Hassel, G. Dumpich and D. von der Linde, Appl. Phys. Lett. 94, 101910 (2009)

[11] A. Hanisch-Blicharski, A. Janzen, B. Krenzer, S. Wall, F. Klasing, A. Kalus, T. Frigge, M. Kammler and M. Horn-von Hoegen, Proceedings of the FEMMS2011- Ultramicroscopy, (in revision) 